\title{
A pilot study of the King LT supralaryngeal airway use in a rural Iowa EMS system
}

\author{
Christopher S. Russi • Michael J. Hartley • \\ Christopher T. Buresh
}

Received: 25 January 2008 / Accepted: 31 March 2008/Published online: 12 June 2008

(C) Springer-Verlag London Ltd 2008

\begin{abstract}
Introduction In 2003, the King Laryngeal Tube (LT) received FDA approval for US sales. Prehospital systems in urban setting have begun evaluating and adopting the LT for clinical airway management. However, it is not routinely approved by State EMS Boards for use by all prehospital providers. Given the LT's simple design there may be benefit to using this tool for airway management in all levels of prehospital providers. This pilot study reviews cases where the King LT was used in a rural Iowa county EMS system. Methods In 2006, the Iowa Department of Public Health / Bureau of EMS approved a 12 month pilot evaluating the King LT by all levels of EMS providers in a rural county EMS system. Following a didactic and competency training session on using the King LT, the providers were instructed to continue airway management per usual protocol but were allowed to use the King LT as a first line airway tool if they felt indicated. Successful placement of airway devices used were determined by colourimetric end-tidal $\mathrm{CO} 2$, chest auscultation and rise as well as vital sign and skin colour improvement. Review of the data was approved by the University of Iowa Institution Review Board (IRB).

Results During the 12-month pilot period, the King LT was used in 13 patients with a mean age of 60.7 years (24-81).
\end{abstract}

\footnotetext{
C. S. Russi $(\square)$

Department of Emergency Medicine, Mayo Clinic, 200 First St SW,

Rochester, MN 55905, USA

e-mail: russi.christopher@mayo.edu

M. J. Hartley $\cdot$ C. T. Buresh

Department of Emergency Medicine, University of Iowa,

Iowa City, IA, USA
}

All patients had cardiopulmonary or traumatic arrest. The King LT was successfully placed on the first attempt in all but one case. The King LT was placed following endotracheal intubation failure in 6/13 (46.1\%) cases and in 3/13 (23.1\%) of cases of Combitube attempt / failure.

Conclusions This small pilot project emphasizes the need for additional rapid airway management tools given the demonstrated ETI failures. The authors believe the King LT has significant potential to impact prehospital airway management as a primary airway device or backup to other failed strategies. Further study is necessary to evaluate the LT's efficacy compared to current strategies.

Keywords Prehospital · Airway

\section{Introduction}

In 2003 the King Laryngeal Tube (King LT $^{\circledR T M}$ ) received 510K clearance from the Food and Drug Administration (FDA) to be marketed in the USA [1]. The original Laryngeal Tube (LT) Airway was introduced to the European anaesthesia market in 1998 by its inventor, VBM of Germany. Following a similar path of other airway tools initially designed for anaesthesia applications, emergency medical services (EMS) systems across Europe and Japan have begun using the LT. The King LT $^{\circledR}$ is a blindly inserted supralaryngeal device that seats in the hypopharynx posterior and inferior to the epiglottis. It has two high-volume low-pressure inflatable balloons, one that occludes the oesophagus and one that inflates in the posterior oropharynx. A ventilation port located between the balloons is positioned over the opening of the trachea (Fig. 1). The balloons create a seal that allows for positive pressure ventilation. In prior controlled settings in the 
Fig. 1 King LT (reproduced with permission from King Systems Corporation)

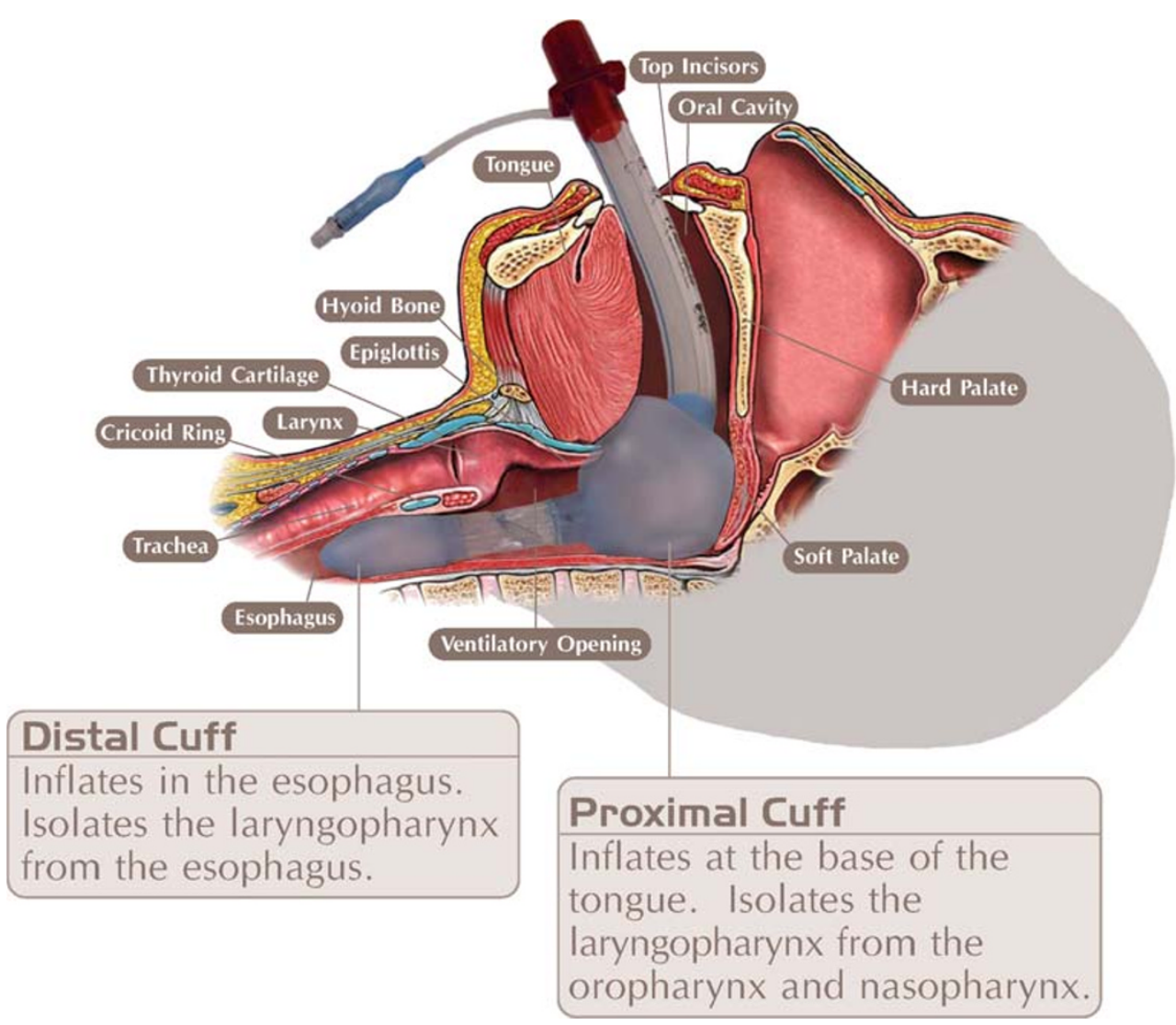

operating suite and mannequin models among anaesthesiologists and paramedic students it has been shown to be both simple and timely to place [2-6]. It has also been found to be an excellent rescue airway device in cases of an unexpectedly difficult airway [7]. However, published data and experience on the prehospital use of the King LT ${ }^{\circledR}$ appear limited. Currently across the USA, the device is not routinely approved for use by all prehospital providers from the basic emergency medical technician (EMT) up through the paramedic level. However, given the King LT ${ }^{\circledR}$ 's simple design requiring less initial and ongoing training, we see potential benefit in using this airway device in both basic and advanced out-of-hospital life support over current airway strategies using the Combitube or endotracheal intubation (ETI). This brief report reviews a series of cases where the King LT $^{\circledR}$ was used by a range of health care providers in the prehospital setting.

\section{Methods}

In 2006 the Iowa Department of Public Health/Bureau of EMS approved a 12-month pilot project evaluating the use of the King LT ${ }^{\circledR}$ by all levels of EMS providers in a rural EMS system. Prior to the genesis of this project, the Iowa EMS Scope of Practice designated the King LT $^{\circledR}$ as a "bridge airway device" to be used only by providers with an EMT-I (Intermediate) certification or above. The chosen pilot EMS system is hospital based and serves a county population of approximately 12,000 persons. The service provides $24 \mathrm{~h}$ of prehospital care and emergency department (ED) assistance in a small critical access hospital. Annual call volume for 2006 was 750 combined basic (BLS) and advanced life support (ALS) transports. System personnel range from EMT-PS [Paramedic SpecialistsNationally Registered Emergency Medical TechnicianParamedic (NREMT-P) equivalent] to EMT-Bs (Basics). Using simulation, medics are annually tested on skills performance for airway management with bag-mask ventilation (BMV) and placement of the endotracheal tube (ETT) and Combitube. Commonly, the service tiers with county first responders and BLS volunteers to provide advanced life support care. However, EMT-PS coverage is not uniform and frequently the highest level of care is the EMT-P (NREMT-I '99 equivalent) and, therefore, limited to medication-assisted intubation without rapid sequence (RSI) capability. Given lengthy transport times and relatively lower patient volumes, the Iowa Department of Public Health/Bureau of EMS approved a countywide pilot project using the King $\mathrm{LT}^{\circledR}$ among all providers as a means for airway management as either a primary device or backup adjunct. 
Medical direction of the service mandated providers approach airway management as per protocol (BMV, ETI, Combitube), but allowed the King LT $^{\circledR}$ to be used first line if felt appropriate by the attending EMS provider. The service was not equipped with end-tidal capnography; therefore placement success was determined by a combination of less reliable means: colourimetric end-tidal $\mathrm{CO}_{2}$, chest auscultation, chest rise as well as vital sign and skin colour improvement.

EMS providers had the three adult FDA approved sizes available for use $(3,4,5)$. A King Systems Corporation representative (King Systems Corp., Noblesville, IN, USA) provided training on the King $\mathrm{LT}^{\circledR}$ to the medical director and training officer who subsequently trained the remaining crew once prior to pilot launch.

Review of the patient and EMS data was approved by the University of Iowa Institutional Review Board (IRB).

\section{Results}

During the 12-month pilot period, the King LT $^{\circledR}$ was placed in 13 patients with a mean age of 60.7 years (range: $24-81$ ); 2 (15.4\%) of the 13 were female. Patients treated were critically ill with either cardiopulmonary or traumatic arrest. Twelve cases were scene responses and one was an inter-facility transfer. The 12 scene calls had a mean en route time of 13.8 min (range: 3-19). In two cases, patient care was passed to an air service and in three other cases patient resuscitation efforts were terminated prehospital. The remaining eight cases resulted in transport to an emergency department with mean transport time of 27.1 min (range: 14-89).

In all cases except one, the King $\mathrm{LT}^{\circledR}$ was placed on the first attempt. Case 3 was the exception noting two King LT $^{\circledR}$ placement attempts. The first King LT $^{\circledR}$ placement failed, as reported in the narrative, due to "jagged patient teeth" lacerating the balloon cuff. This patient underwent multiple ETI attempts with success noted on the fifth. During transport, the ETT was recognized to have been dislodged and following a failed Combitube attempt, the second King LT $^{\circledR}$ attempt occurred en route in the back of the ambulance.

The King $\mathrm{LT}^{\circledR}$ was placed following intubation failures in $6(46.1 \%)$ of the 13 cases and in 3 cases $(23.1 \%)$ following Combitube placement attempt/failure. In case 6 the King LT $^{\circledR}$ was used following the inability to ventilate using a Combitube. After close review of this case, we felt this was not secondary to Combitube failure or operator error. This particular case was a traumatic arrest with bilateral pneumothoraces requiring subsequent bilateral needle decompression. EMS noted difficult ventilation with the Combitube and the King $\mathrm{LT}^{\circledR}$ was placed following

Table 1 Case demographics

\begin{tabular}{|c|c|c|c|c|c|c|c|c|c|}
\hline \multirow[b]{2}{*}{$\begin{array}{l}\text { Case } \\
\text { No. }\end{array}$} & \multirow[b]{2}{*}{ Age } & \multicolumn{2}{|l|}{ Time (min) } & \multirow[b]{2}{*}{ Aetiology } & \multirow[b]{2}{*}{ Provider } & \multicolumn{3}{|c|}{ Attempt(s) } & \multirow[b]{2}{*}{$\begin{array}{l}\text { How King } \\
\text { LT }^{\circledR} \text { used }\end{array}$} \\
\hline & & $\begin{array}{l}\text { En route } \\
(n=13)\end{array}$ & $\begin{array}{l}\text { Scene to } \\
\operatorname{ED}(n=8)\end{array}$ & & & ETI & Combitube & $\begin{array}{l}\text { King } \\
\mathrm{LT}^{\circledR}\end{array}$ & \\
\hline 1 & 51 & 17 & 20 & Traumatic arrest & EMT-P & 0 & 0 & 1 & First line \\
\hline 2 & 36 & 14 & 16 & Cardiac arrest & EMT-P & 1 & 1 & 1 & Rescue for ETI \\
\hline 3 & 79 & 0 & 89 & $\begin{array}{l}\text { CVA with resp. } \\
\text { arrest }\end{array}$ & EMT-PS & 5 & 1 & 2 & Rescue for ETI \\
\hline 4 & 54 & 11 & 15 & Traumatic arrest & EMT-PS & 1 & 0 & 1 & Rescue for ETI \\
\hline 5 & 62 & 10 & Air service tier ${ }^{a}$ & MVC - unresponsive & EMT-PS & 1 & 0 & 1 & Rescue for ETI \\
\hline 6 & 62 & 19 & No transport & Traumatic arrest & EMT-P & 0 & 1 & 1 & Rescue for Combitube \\
\hline 7 & 67 & 20 & 14 & $\begin{array}{l}\text { Resp. distress- } \\
\text { unresponsive }\end{array}$ & EMT-P & 2 & 0 & 1 & Rescue for ETI \\
\hline 8 & 69 & 3 & No transport & Cardiac arrest & EMT-B & 0 & 0 & 1 & First line \\
\hline 9 & 55 & 16 & 19 & Cardiac arrest & EMT-B & 0 & 0 & 1 & First line \\
\hline 10 & 81 & 15 & 23 & Cardiac arrest & EMT-B & 0 & 0 & 1 & First line \\
\hline 11 & 67 & 15 & 21 & Cardiac arrest & EMT-PS & 0 & 0 & 1 & First line \\
\hline 12 & 24 & 12 & No transport & Traumatic arrest & EMT-P & 0 & 0 & 1 & First line \\
\hline 13 & 80 & 14 & Air service tier ${ }^{\mathrm{a}}$ & Unresponsive & EMT-P & 3 & 0 & 1 & Rescue for ETI \\
\hline Mean & 60.5 & 13.8 & 27.13 & & & & & & \\
\hline Median & & 14.0 & 19.5 & & & & & & \\
\hline IQR & & $\begin{array}{l}5.0 \\
(11.0-16.0)\end{array}$ & $\begin{array}{l}5.75 \\
(15.75-21.5)\end{array}$ & & & & & & \\
\hline
\end{tabular}

EMT-P Iowa Paramedic (National EMT-Intermediate equivalent), EMT-PS Iowa Paramedic Specialist (National EMT-Paramedic equivalent), ETI endotracheal intubation, $I Q R$ interquartile range

${ }^{a}$ Patient transported by air EMS from scene (no transport by ground EMS) 
needle decompression. Combitube failures were the impetus for King LT $^{\circledR}$ placement in other cases as well. Case 2 documented absent breath sounds using the Combitube placed by first responders leading to an ETI attempt with subsequent inability to visualize the vocal cords. A King LT $^{\circledR}$ was placed following the failed ETI attempt without difficulty with resultant patient "colour improvement" and "adequate chest rise" (Table 1).

\section{Discussion/limitations}

The results from this pilot project provide no specific statistical comparative significance, but reinforce the concerning issue of prehospital endotracheal intubation potentially leading to poor patient outcomes [8]. The majority of prehospital studies citing poor outcomes or difficulties with ETI and RSI are based on urban EMS systems with presumed larger patient volumes and experience for providers [9-13]. Serving smaller patient populations and often having limited resources, funding and training puts the rural EMS provider at a greater disadvantage for experience and the tools to maintain proficiency with endotracheal intubation [14, 15].

Limitations to this pilot do not allow for specific comment on or validation of the efficacy of the King LT ${ }^{\circledR}$ as a first or second line airway tool. No digital end-tidal $\mathrm{CO}_{2}$ monitoring was available; nearly all of the patients were critically ill or near dead and no patient follow-up was available for the few who survived to the ED.

This pilot also does not allow for a direct comparison between the Combitube and the King LT $^{\circledR}$. One case used the King LT $^{\circledR}$ following a Combitube insertion; however, the authors feel this is not secondary to Combitube failure but rather physiological alterations limiting the Combitube's ability to ventilate (bilateral pneumothoraces). Had the King LT $^{\circledR}$ been placed first the presumption is that it too would have failed (case 3 failure).

Anecdotally via the EMS report narratives, patients were easy to ventilate using the King LT $^{\circledR}$ with "adequate chest rise" and "bilateral breath sounds" noted in 10 of the 13 cases $(76.9 \%)$. Two of the three remaining cases did not have lung sounds documented and one documented "no sounds" with "copious blood coming from the King LT $^{\circledR}$..." following a traumatic arrest. With the exception of one case, the King LT $^{\circledR}$ was placed without difficulty using one attempt.

A single lumen, single port balloon inflation design eliminates the potential difficulties providers may experience using a Combitube when not practiced or proficiency maintained $[4,16]$. The device is blindly inserted and is currently available in three approved sizes. Choice of King $\mathrm{LT}^{\circledR}$ size is straightforward and determined by patient height.

\section{Conclusion}

Although this pilot project is small, it emphasizes the need for additional rapid airway management adjuncts given the number of ETI failures. From the above case narratives, we feel this device has significant potential to impact prehospital airway management, either as a primary device or as backup to difficult or failed ETI. Further prospective trials are needed to validate the efficacy of the device compared to current management strategies (ETI and Combitube).

\section{References}

1. Administration, U.S.F.a.D., King-LT ${ }^{\circledR}$ FDA marketing approval, 9 January 2003, Rockville, MD

2. Hagberg C, et al (2006) An evaluation of the insertion and function of a new supraglottic airway device, the King $\mathrm{LT}^{\circledR}$, during spontaneous ventilation. Anesth Analg 102(2):621-625

3. Komatsu $\mathrm{R}$ et al (2005) Comparison of the intubating laryngeal mask airway and laryngeal tube placement during manual in-line stabilisation of the neck. Anaesthesia 60(2):113-117

4. Russi CS, Miller L, Hartley MJ (2008) A comparison of the KingLT $^{\circledR}$ to endotracheal intubation and Combitube in a simulated difficult airway. Prehosp Emerg Care 12(1):35-41

5. Kurola J et al (2006) Comparison of airway management with the intubating laryngeal mask, laryngeal tube and CobraPLA by paramedical students in anaesthetized patients. Acta Anaesthesiol Scand 50(1):40-44

6. Russi CS, Wilcox CL, House HR (2007) The laryngeal tube device: a simple and timely adjunct to airway management. Am J Emerg Med 25(3):263-267

7. Matioc AA, Olson J (2004) Use of the Laryngeal Tube in two unexpected difficuLT ${ }^{\circledR}$ airway situations: lingual tonsillar hyperplasia and morbid obesity. Can J Anaesth 51(10):1018-1021

8. Wang HE et al (2004) Out-of-hospital endotracheal intubation and outcome after traumatic brain injury. Ann Emerg Med 44(5):439 450

9. Davis DP et al (2004) The impact of hypoxia and hyperventilation on outcome after paramedic rapid sequence intubation of severely head-injured patients. J Trauma 57(1):1-8; discussion 8-10

10. Davis DP et al (2003) The effect of paramedic rapid sequence intubation on outcome in patients with severe traumatic brain injury. J Trauma 54(3):444-453

11. Davis DP et al (2005) The impact of prehospital endotracheal intubation on outcome in moderate to severe traumatic brain injury. J Trauma 58(5):933-939

12. Murray JA et al (2000) Prehospital intubation in patients with severe head injury. J Trauma 49(6):1065-1070

13. Bochicchio GV et al (2003) Endotracheal intubation in the field does not improve outcome in trauma patients who present without an acutely lethal traumatic brain injury. J Trauma 54(2):307-311

14. Burton JH et al (2003) Endotracheal intubation in a rural EMS state: procedure utilization and impact of skills maintenance guidelines. Prehosp Emerg Care 7(3):352-356

15. Wang HE et al (2005) Defining the learning curve for paramedic student endotracheal intubation. Prehosp Emerg Care 9(2):156-162

16. McManus JH, Arkava G, Bose T, Schwartz S, Ludwig RD (2005) Combitube dual-lumen esophageal airway device retention skills in deployed army combat medics. Acad Emerg Med 12(5 Suppl 1): $162 \mathrm{a}$ 